\title{
sur la gestion et les finances publiques En Septembre 2020
}

\section{BUDGeT DE L'ÉTAT ET DES OPÉRATEURS}

\section{$\lambda$ Politiques budgétaires}

Le gouvernement lance le plan de relance Le contenu du plan de relance a été annoncé le 3 septembre et fait l'objet d'une brochure détaillée. II équivaut à $100 \mathrm{Md}$ dont $40 \mathrm{Md} €$ de fonds européen. Les 70 mesures se répartissent entre les axes « écologie » (30 Md€), « compétitivité » (34 Md€) et « cohésion » (36 Md€). Ce plan se matérialise à travers plusieurs instruments : la troisième loi de finances rectificative pour les moyens engagés en 2020, le projet de loi de finances pour 2021 et le programme d'investissement d'avenir, le PLFSS 2021 et des mesures de simplification réglementaires ou législatives.

L'avis du HCFP sur le PLF 2021 et le PLFSS 2021

Dans un avis $n^{\circ} 2020-5$ du 21 septembre sur le PLF 2021 et PLFSS 2021, le Haut conseil des finances publiques (HCFP) estime que les prévisions macroéconomiques du gouvernement sont, dans l'ensemble, " plausibles» ou «prudentes». Il est en revanche plus circonspect à propos de la cohérence entre le PLF et la loi de programmation des finances publiques du 22 janvier 2018 qui est désormais une "référence dépassée ». II estime ainsi nécessaire l'adoption d'une nouvelle LPFP dès le printemps 2021.

Le projet de loi de finances 2021 : un contexte très particulier

Le projet de loi de finances pour 2021, présenté le lundi 28 septembre en conseil des ministres, est marqué par le contexte économique lié à la crise du Covid et le financement du plan de relance. Le déficit public s'établit à -6,7\% du PIB (-10,2 \% en 2020). Le solde de l'État s'établirait à 152, 8 Md€ en 2021 ( 195,2 Md€ en 2020). La dette publique décroîtrait pour s'élever à 116,2\% du PIB même si les émissions de dette connaîtront en 2021 un niveau record (260 MdE). Les dépenses publiques s'élèveraient en 2021 à $58,5 \%$ du PIB (hors crédits d'impôts)(62,8\% en 2020). L'État est le principal financeur du plan de relance (86 Md€ sur $100 \mathrm{Md}$ ). La mission budgétaire spécifique financera $36 \mathrm{Md} €$ en autorisations d'engagement et $22 \mathrm{Md} €$ de crédits de paiement dès 2021. En outre, le budget se veut « volontariste » en termes de dépenses. Au niveau des ministères, parmi les gagnants, on trouve notamment la mission justice (+150 M) ainsi que l'éducation nationale (+100 M). Les effectifs de fonctionnaires sont stabilisés (-157 emplois avec de grandes disparités entre les ministères : -2.163 aux Finances(dont 1800 à la DGFiP qui a déjà subi 38500 suppressions d'emplois ente 2004 et 2019) au profit de la Justice, de l'Intérieur, des Armées et des services du Premier ministre). La politique de baisse de la pression fiscale (45 Md€ de baisses entre 2018 et 2021) n'est cependant pas remise en cause et même poursuivie : baisse de l'impôt sur les sociétés, suppression progressive de la taxe d'habitation et baisse des impôts de production (10 Md€ en 2021).

\section{L'évaluation verte du budget de l'État}

Un document ayant pour objectif d'évaluer « l'impact environnemental du budget de l'État» a été publié pour la première fois en annexe au PLF. La première partie présente l'impact environnemental des crédits budgétaires et des dépenses fiscales. Le PLF comporterait par rapport à 2020, une augmentation de $+29 \%$ des mesures favorables à l'environnement. Elles atteignent, 38, 1 Md€ dans PLF 2021 contre 29,6Md€ en LFI 2020. Parmi ces mesures «favorables », on note celles liées aux énergies renouvelables (6,9 Md€), certains crédits du Plan de relance (6,6 Md€), les aides publiques au développement (1,9 Md€), les taxes affectées aux agences de l'eau. Les dé- 
penses «brunes » du budget, c'est-à-dire nocives à l'égard des objectifs environnementaux sont estimées à $10 \mathrm{Md} €$ et sont essentiellement des niches fiscales.

\section{Dette publique}

Hausse record de la dette publique au deuxième trimestre

Une note d'information rapide de l'INSEE du 25 septembre indique qu'à la fin du deuxième trimestre 2020, la dette publique s'établit à 2 638,3 Md€, en hausse de 199, 9 Md€ par rapport au trimestre précédent. L'institut estime qu'exprimée en pourcentage, cette hausse de 12,7 points est la plus forte augmentation trimestrielle depuis que I'INSEE publie cet indicateur soit depuis 1995.

\section{त Patrimoine de l'État}

Le rapport de la commission d'enquête sur les autoroutes

Tout comme l'avis de l'Autorité de la concurrence de 2014 et plusieurs rapports successifs de la Cour des comptes, le rapport sénatorial de la commission d'enquête sur le contrôle, la régulation et l'évolution des concessions autoroutières, publié le 16 septembre, reconnaît certains progrès en matière de régulation et du contrôle du secteur autoroutier, notamment suite à la création de l'ARAFER devenue Autorité de régulation et de transport (ART) et à un contrôle renforcé des marchés passés par les sociétés concessionnaires dont les entreprises «liées ». Le rapport invite cependant le pouvoir exécutif à réfléchir à la gestion des autoroutes à l'échéance des concessions historiques. Selon le document, «si le modèle concessif était conservé, il faudrait des clauses de revoyures tous les cinq ans, permettant de réviser les tarifs ou la durée de la concession en cas de surentabilité ». Parallèlement, le rapport préconise d'établir une définition claire de la notion de "besoins de la concession" afin d'empêcher les sociétés concessionnaires d'autoroutes de soustraire à leurs obligations de publicité et de mise en concurrence pour la passation de certains de leurs marchés.

\section{$\pi$ Budget des opérateurs}

La Caisse des dépôts présente son plan de relance

La Caisse des dépôts (CDC) a annoncé le 7 septembre son propre plan de relance à hauteur de $26 \mathrm{Md}$. 6,3Md€ seront consacrés à la transition écologique, 8,3 Md€ pour les entreprises de tous les secteurs, $500 \mathrm{M} €$ pour soutenir les secteurs de la santé et du médico-social, 11,1 Md€ pour accélérer la construction des logements. La plupart de ces investissements concernent la vie concrète des français. Par exemple, dans le cadre de la transition écologique, un des objectifs est de permettre l'installation de 50000 bornes électriques de recharge à travers le territoire pour inciter les français à utiliser des voitures propres.

\section{FINANCES LOCALES}

\section{入 Données générales sur les finances locales}

En 2019 : fonctionnement modéré, investissement dynamique

L'Observatoire des finances et de la gestion publique locale (OFGPL) a rendu son traditionnel rapport sur les finances locales. II ressort de ce document que les administrations publiques locales affichent un léger besoin de financement en 2019 après avoir été en excédent en 2018 (-0,9 Md€ après +2,7 Md€ en 2018). Cette situation est tout à fait normale en fin de cycle électoral avec la hausse des dépenses d'investissement (+13\% en 2019 après $+5,2 \%$ en2018). La dette atteint 210,3 Md€, soit une hausse de +4,6 Md€, sauf pour les départements, qui voient leur endettement diminuer de 0,9 Md€. En termes de dépenses de fonctionnement, celles-ci n'ont augmenté que $+1,1 \%$, après $+0,3 \%$ en 2018 s'inscrivant dans la trajectoire de la loi de programmation des finances publiques 2018-2022. L'épargne brute a progressé en 2019 beaucoup plus rapidement qu'en 2018 ( $+11,0 \%$ après $+5,6$ $\%)$. Les recettes $d$ 'investissement (FCTVA, subventions reçues et dotations à l'investissement) ont, elles aussi, très fortement progressé (+7,9\%) et l'encours de dette s'est une nouvelle fois stabilisé en 2019, constat atypique pour des années aussi dynamiques en investissements.

\section{Conjoncture : le secteur local résilient en 2020}

La note de conjoncture de la Banque Postale du 24 septembre rappelle l'effet ciseaux de la crise sanitaire avec des dépenses de fonctionnement plus élevées et des ressources en forte baisse. L'épargne brute se replie de 18,1\% à 32,2 Md€ et les dépenses d'investissement pourraient diminuer de 5,8\%. En 2020, les dépenses de fonctionnement des collectivités locales resteraient modérées (+1,4\%, pour atteindre 191,9 Md€, soit un ralentissement par rapport à 2019; mais $+2,2 \%$ en intégrant le transfert du financement de l'apprentissage aux branches professionnelles (- 1,6 Md€ de dépenses pour les régions) ; mais, 
encore, $+1,2 \%$ sans les dépenses imprévues engagées par les collectivités locales au titre de la crise sanitaire). Les recettes de fonctionnement ralentiraient de $2,0 \%$, soit une perte de 4,5 Md€ imputable principalement à la fiscalité, aux produits de la tarification mais également pour près de moitié par la recentralisation du financement de l'apprentissage. Le produit des impôts et taxes, hors impact de la recentralisation de I'apprentissage, diminuerait de 0,8 \% (- 2,1 \% y compris recentralisation), soit une contraction de 1,2 Md€.

De grandes disparités entre les territoires urbains

France urbaine (Portrait financier $n^{\circ} 5$, septembre 2020) livre une intéressante étude sur la situation financière des territoires urbains (80 communautés d'agglomération de plus de 150000 habitants en métropole). Le résultat de cette analyse aboutit à une progression globale des dépenses $d^{\prime}$ investissement de $+16,6 \%$ pour les communes et $+14,4 \%$ pour les groupements, mais avec de profondes disparités entre les territoires. Ainsi, 12 territoires urbains connaissent une baisse de leurs investissements. En termes de recettes de fonctionnement, celles-ci représentent $91 \%$ des ressources des territoires urbains en 2019, soit 71,7 milliards d'euros. La hausse s'explique principalement par la revalorisation des bases.

\section{入 Transfert de l'État}

Montant de la répartition de la dotation dite " Natura 2000 "

L'article 252 de la loi de finances pour 2020 a institué à compter de 2020 une dotation budgétaire destinée aux communes de moins de 10000 habitants, dont le territoire fait partie pour une proportion significative d'un site « Natura 2000 » ou d'un cœur de parc national ou est situé dans un parc naturel marin et sous réserve du respect de conditions de population, de potentiel fiscal et de superficie. La première répartition de cette dotation porte sur un montant total de 10 millions d'euros en 2020.

\section{$\lambda$ Fiscalité locale}

L'AMF critique les choix de baisse des impôts économiques

Dans un communiqué du 2 septembre, l'Association des maires de France exprime clairement son désaccord avec les éléments fiscaux du plan de relance. L'AMF estime que le gouvernement effectue un remplacement de recettes fiscales locales par des dotations d'État, notamment dans le cadre de la réforme des impôts de production. En effet, l'effet du plafonnement amélioré de la contribution économique territoriale (CET) et l'allègement de fiscalité pour certaines industries imputera les ressources financières locales qui seront remplacées par des dotations. Ce faisant, les communes et EPCI perdent une nouvelle fois une part de levier fiscal.

\section{FinANCES SOCIALES}

\section{$\pi$ Situation des comptes sociaux}

Protection sociale des indépendants : des iniquités subsistent

France Stratégie a publié le 22 septembre le rapport du HCFiPS commandé fin 2019 par le Premier Ministre sur la protection sociale des non-salariés. Le rapport constate la persistance d'inéquités entre salariés et indépendants, notamment au regard des conditions d'assiette; il relève que le développement de l'économie collaborative pose question au regard du rattachement aux régimes de Sécurité sociale et prend acte de la normalisation de la situation après la crise de l'interlocuteur social unique (ISU). Enfin il constate que les contrôles sont encore insuffisamment développés et recommande une meilleure articulation avec l'administration fiscale.

\section{Commission des comptes de la Sécurité} Sociale : déficit de $47 \mathrm{Md} €$

La Commission des comptes de la SS a présenté le 29 septembre son rapport pour les exercices 2020 et 2021. Elle anticipe un déficit global du Régime général et du FSV sans précédent de -46,6 Md€ (légèrement moindre toutefois que la prévision de juin à -52 Md€ du fait d'une contraction moins marquée qu'anticipé de la masse salariale), en aggravation de près de $50 \mathrm{Md}$ f par rapport au résultat de 2019. La branche maladie enregistrerait un déficit de $-30 \mathrm{Md} €$, la branche accidents du travail de -0,3 Md€, la branche famille de -3,3 Md€, la branche vieillesse de -7,9 Md€ et le FSV de-3,2 Md€. Cette situation s'explique d'abord par la baisse de $-4,2 \%$ des recettes des régimes de base et du FSV et en tout premier lieu des cotisations sociales $(-7,6 \%) d u$ fait du recours à l'activité partielle et des reports de paiement pour les travailleurs indépendants, de $-5,5 \%$ des rentrées de CSG et de $-2,5 \%$ des rentrées des impôts et taxes affectées (ITAF). Parallèlement, la progression des dépenses sera de $3,7 \%$, les dépenses de la santé progressant de $5,8 \%$ du fait des dépenses exceptionnelles de 15 Md€ occasionnées par la crise du COVID-19. Ces dépenses se répartissent en 4,8 Md€ pour 
I'achat de masques, de matériels et produits pour les tests, en 5,5 Md€ pour les indemnités journalières, les tests l'aide aux professionnels de santé ayant subi des pertes d'activité et 4,7 Md€ pour le financement des établissements sanitaires et médico-sociaux (dont les premières mesures indemnitaires pour les personnels, mais hors mesures du «Ségur de la Santé »). De ce fait I'ONDAM 2020 aura progressé de $7,6 \%$, niveau sans précédent, contre $2,6 \%$ en 2019. Pour 2021, le déficit du Régime général et du FSV, avant toute prise en compte des mesures nouvelles du PLFSS 2021 (cf infra), serait ramené à -25,5 Md€, légèrement inférieur à celui du dernier record historique de 2010. II est escompté un fort rebond des recettes (+5,9\%) grâce à la reprise de l'activité et un retour à la normale de la progression des dépenses (+3,3\%), La progression tendancielle de I'ONDAM serait ramenée à 1,4\%, avant prise en compte des mesures nouvelles du PLFSS; une provision de 4,3 Md€ est prévue pour la crise sanitaire (principalement pour les tests et l'acquisition de stocks de vaccins). Ces prévisions sont fondées sur l'hypothèse d'une absence de confinement généralisé.

\section{$\pi$ Lois de financement de la sécurité sociale}

PLFSS pour 2021: le déficit restera supérieur à 20Md€ jusqu'en 2024

Les Ministres des Solidarités et de la Santé et des Comptes publics ont présenté à la presse le PLFSS 2021 le 29 septembre. II accuse un déficit global du RG+FSV de -27 Md€, avec une trajectoire qui ne prévoit pas de déficit inférieur à 20 Md€ jusqu'en 2024. L'une des mesures phares en est la création de la $5^{\text {e }}$ branche de la SS pour le soutien à l'autonomie, qui a été prévue par la loi du 7 août 2020 (cf. REPÈRES d'août). II sera présenté officellement au premier conseil des ministres d'octobre.

\section{$\lambda$ Politiques d'allègement des charges et coût du travail}

\section{Les volets sociaux du plan de relance}

Le Premier Ministre a présenté le 3 septembre le plan de relance de $100 \mathrm{Md}$. Le volet social, dont une partie avait été déjà annoncée(cf Repères de juin et juillet), concerne d'abord la sauvegarde de l'emploi : 7,6 Md€, dont 6,6 Md€ sont consacrés au nouveau dispositif $d^{\prime}$ " activité partielle de longue durée » en place depuis le 1er juillet et $1 \mathrm{Md} €$ à l'abondement du FNE pour financer l'accompagnement en formation des transitions professionnelles. Près de $7 \mathrm{Md}$ Łont à l'emploi des jeunes, avec en particulier la prime à l'embauche (1,1 Md€), l'aide à l'apprentissage et à la professionnalisation (2,7 Md€) et la formation des jeunes aux métiers d'avenir (1,6 Md€). Environ $2 \mathrm{MdE}$ visent à renforcer les moyens d'intervention des opérateurs de l'emploi (France Compétences et Pôle Emploi, pour $1 \mathrm{Md}$ ) et à financer la digitalisation de la formation (0,9 Md€). Dans le domaine de la santé et de la dépendance, on retrouve les $6 \mathrm{Md} €$ que le Ségur de la Santé avait annoncés pour l'investissement public dans le secteur hospitalier. Enfin 0,8 MdE vont au soutien des personnes précaires (majoration de l'allocation de rentrée scolaire et ticket de RU à $1 €$, développement de l'hébergement d'urgence). Le reste des financements est pour l'essentiel consacré à la recherche ( $3 \mathrm{Md}$ et à la cohésion territoriale $(9,5 \mathrm{Md})$.

\section{L'État s'apprête à ponctionner Action Lo-} gement en 2020 et 2021

Selon des informations parues dans les Echos du 7 septembre, le Gouvernement s'apprête à faire une nouvelle ponction sur la trésorerie $d^{\prime}$ " Action Logement », organisme qui a remplacé le $1 \%$ logement pour la collecte de la Participation des employeurs à l'effort de construction-PEEC $(0,45 \%$ sur la masse salariale). En 2019, le gouvernement avait déjà prélevé 0,5 Md€ sur la trésorerie d'Action Logement, il serait envisagé une nouvelle ponction de $1 \mathrm{Md} €$, voire une réduction du taux de la PEEC

Le CICE aura créé un peu plus de 100000 emplois en 5 ans

v. infra Fiscalité

\section{त Dépenses de santé / Hôpital / Assurance maladie}

\section{Dérapage des dépenses de santé en 2020}

Les achats massifs de matériels sanitaires (masques, respirateurs ...) au printemps pendant la première vague de la crise sanitaire, puis les premiers effets sur 2020 des revalorisations salariales du Ségur de la santé (cf Repères de juillet), auront majoré de plus de $8 \mathrm{Md}$ les dépenses de santé prises en compte dans I'ONDAM. Vont s'y ajouter la croissance exponentielle depuis l'été des tests de dépistage, dont le surcoût pourrait s'élever à 1,3 Md€ d'ici la fin de l'année. De ce fait, le dérapage de l'ONDAM pourrait passer de la croissance initialement prévue de $2,5 \%$ à 7 ou 7,5\% d'ici décembre. A ce stade, il n'y a pas eu de dépôt d'un PLFSSR rectificatif pour 2020. 
Moins de $7 \%$ des dépenses de soins des Français reste à leur charge

L'édition annuelle des dépenses de santé pour 2019 publiée le 15 septembre par la DREES fait apparaître la poursuite de la baisse de la participation financière des ménages à leurs dépenses de santé, à 6,9\% (contre $7 \%$ en 2018). La part de la SS progresse légèrement ( de 78 à 78,2\%), alors que celle des complémentaires santé reste stable. Au total, en France, la dépense de santé s'établit à $11,3 \%$ du PIB, soit 1,4 points de plus que la moyenne de l'OCDE.

\section{\ Assurance chômage}

Les pistes pour apurer le déficit de l'Assurance chômage

Le bureau de I'UNEDIC du 9 septembre a examiné diverses pistes pour remettre le régime sur les rails après la prévision de déficit abyssal pour 2021 (-25,7 Md€) : révision des conditions de prise en charge d'un certain nombre de dépenses par le régime, augmentation de la cotisation patronale (actuellement fixée à 4,05\% du salaire), augmentation de la part de la CSG attribuée à I'UNEDIC depuis la suppression des cotisations salariales en 2018, ; pour la dette (50 Md€ à la fin de 2020), la piste d'un transfert à la CADES, dont les missions pourraient être étendues à cette fin pourraient être examinées.

Maintien du niveau de reste à charge pour l'activité partielle de longue durée

Le gouvernement a finalement décidé de maintenir à $15 \%$ le reste à charge de l'employeur pour I'Activité partielle de longue durée (APLD v. Repères de juin) , y compris pour les accords signés après le 31 octobre 2020 alors qu'il prévoyait de le porter à $20 \%$ à cette date parce qu'il voulait inciter les entreprises à entrer rapidement dans le nouveau dispositif. Le Ministère du Travail, selon les Echos, prévoit que 500000 salariés seraient couverts par l'APLD en 2021, soit un même effectif que pour le chômage partiel de droit commun qui est nettement moins avantageux ; à ce stade, le coût total de l'activité partielle serait de 6,6 Md€ pour 2021.

225 millions d'euros de fraude au chômage partiel

Selon les informations du Ministère du Travail diffusée le 7 septembre, la fraude au dispositif de chômage partiel représenterait 225 millions d'euros sur les $30 \mathrm{Md}$ engagés à ce titre depuis le début du confinement jusqu'à septembre, sur un nombre de salariés bénéficiaires qui est passé de 8,8 millions en avril à 2,4 millions en juillet.
27000 opérations de contrôle ont été effectuées a posteriori, permettant de soupçonner près de 10000 fraudes.

\section{$\lambda$ Formation professionnelle}

La réforme de la formation professionnelle va coûter plus cher que prévu

Le dérapage du coût de la réforme prévue par loi du 5 septembre 2018, a été mis en lumière par un récent rapport conjoint de l'IGF et de I'IGAS. Alors que le besoin de financement lié à la reprise du financement des contrats d'apprentissage conclus avant le 31 décembre 2019 par France Compétences était initialement estimé à $-2,5 \mathrm{MdE}$, le rapport conclut à résultat comptable négatif de l'opérateur central de -5,4 Md€. Compte-tenu de la dynamique des dépenses induite par le succès de la réforme (mais sans anticiper les effets de la crise liée au COVID 19), la situation devrait perdurer. Le rapport propose plusieurs pistes pour un retour à l'équilibre.

\section{$\pi$ Solidarité / Lutte contre la pauvreté / Revenu universel}

Les minima sociaux contribuent à réduire le taux de pauvreté

L'enquête annuelle de la DREES « minima sociaux et prestations sociales », publiée le 22 septembre, confirme pour 2017 les résultats constatés en 2016, à savoir que les prestations sociales et la fiscalité directe diminuent le taux de pauvreté de 8 points : $14,1 \%$ de la population est pauvre, contre $22,1 \%$ si la redistribution sociale et fiscale n'existait pas. Cette redistribution étant plus particulièrement marquée pour les familles monoparentales, les personnes de moins de 20 ans et les personnes en situation de handicap et de perte d'autonomie. L'étude constate par ailleurs que le nombre d'allocataires d'au moins un minimum social (4,25 millions fin 2018) a légèrement augmenté en $2018(+0,6 \%)$ alors qu'il avait diminué en 2017 et surtout en 2016.

\section{入 Autonomie / Dépendance}

\section{Le rapport de I'IGF -IGAS sur la future branche autonomie}

Dans la perspective de la création de la cinquième branche " autonomie » actée par la loi du 7 août 2020 relative à la dette sociale et à l'autonomie » (cf. Repères d'août), la mission conjointe conduite par Laurent Vachey a remis son rapport au gouvernement le 14 septembre. S'agissant du périmètre de la future $5^{\mathrm{e}}$ branche, le rapport préconise d'en élargir le périmètre au-delà de celui 
actuel de la CNSA qui a été désignée comme son futur gestionnaire, en incluant des prestations relevant aujourd'hui d'autres branches (allocation d'éducation de l'enfant handicapé, une part des dépenses d'unités de soin de longue durée...) ou du budget de l'État ( allocation aux adultes handicapés, aides aux postes aux travailleurs handicapés....) ce qui ferait passe le champ de la branche de $27 \mathrm{Md}$ à $41 \mathrm{Md}$. Pour la gouvernance, celle-ci resterait dans le cadre de l'organisation de la CNSA, mais avec une contractualisation des relations avec les autres acteurs (départements et ARS) et des moyens de pilotage accrus. Les propositions les plus débattues portent sur le financement, dans l'objectif fixé par le gouvernement de mobiliser des ressources supplémentaires pour le grand âge de $1 \mathrm{Md}$ en 2021 à 3,5 Md€ en 2024 : transferts du FFR, prélèvements sur Action Logement, réaffectation d'économies sur l'AAH et sur l'APA, réduction du champ de certaines niches sociales et fiscales (exonération des cotisations patronales pour l'emploi de personnel à domicile...), mobilisation des patrimoines immobiliers, et surtout de nombreuses propositions en matière fiscale : alignement du taux normal de la CSG sur les pensions $(8,3 \%)$ sur celui des revenus d'activité $(9,2 \%)$, suppression de l'abattement de $10 \%$ de l'IR sur les pensions, augmentation des droits de transmission.

\section{$\lambda$ Contrôles / Lutte contre la fraude sociale}

Un nouveau rapport du Sénat sur la fraude aux prestations sociales

La Cour des Comptes a rendu le 9 septembre les conclusions de l'enquête sur les fraudes aux prestations sociales que lui avait commandé la Commission des Affaires sociales du Sénat en novembre 2019, enquête qui couvre toutes les branches du régime général de la Sécurité Sociale, Pôle Emploi et I'AGIRC-ARRCO, soit $521 \mathrm{Md}$. La Cour n' a pas voulu faire d'estimation du coût global de la fraude, seule la CNAF se livrant à une telle évaluation (En 2018, un montant de fraude -détectée ou non- s'élevant à 2,3 Md€, soit 3,2 \% du montant des prestations, portant principalement sur le RSA-prestation de loin la plus fraudée, la prime d'activité et les aides au logement). Elle salue les efforts réalisés en matière de contrôles, en particulier par la branche famille et la branche maladie. La Cour propose 15 recommandations axées autour de 3 chantiers : $\left.1^{\circ}\right)$ sécuriser les identifications des bénéficiaires, par croisement des fichiers, notamment avec ceux de la DGFiP (Ficoba) et par un recours accru à l'identification physique ; 2) fiabiliser les salaires, les ressources et les situations professionnelles en généralisant l'usage du Dispositif de Ressources Mutualisés que va mettre en place la CNAF à partir du début 2021 pour le paiement des aides au logement ; $3^{\circ}$ ) gérer de façon plus rigoureuse les droits et facturations de frais de santé à l'Assurance maladie. La Cour propose ensuite de renforcer l'efficacité des contrôles et des sanctions, notamment à l'égard de professionnels de santé délictueux. Parallèlement, la Commission des Finances de l'Assemblée Nationale déposait son rapport d'information sur l'application de la loi du 23 octobre 2018 relative à la lutte contre la fraude.

\section{FISCALITÉ ET PROCÉDURE FISCALE}

\section{$\pi$ Conrôle fiscal}

Un rapport sur le contrôle fiscal : évaluation difficile et stratégie à clarifier...

Un rapport sénatorial portant sur l'adéquation des moyens humains et matériels aux enjeux du contrôle fiscal, rédigé par les sénateurs Nougein (LR) et Carcenac (PS), rapporteurs spéciaux de la mission «Gestion des finances publiques et des ressources humaines " de la commission des finances du Sénat (art. 57 LOLF), repose sur trois constats initiaux : la diminution des résultats du contrôle fiscal depuis 2015 hors exercice 2019 (11,3 Md€ en 2019 contre 8,7 Md€ en 2018) ; le développement de techniques innovantes d'analyse de données; la fin de la sanctuarisation des effectifs dédiés au contrôle fiscal. Comme la Cour des comptes (cf. Repères déc. 2019), il souligne les difficultés d'une appréciation des résultats du contrôle fiscal dont la variation dépend des contentieux fiscaux à enjeux, dont le Parlement est peu informé. L'absence d'évaluation de la fraude fiscale, alors que les administrations fiscales étrangères y parviennent, ne permet pas un pilotage efficace. Enfin, ce rapport vient surtout souligner les résultats décevants apportés par les nouveaux outils technologiques en matière de contrôle fiscal. En 2019, sur 12 Md€ mis en recouvrement après contrôles fiscaux, seulement 785 M€ ont été recouvrés grâce au data mining (exploration de données) alors que le nombre de dossiers, issus d'un traitement des données au sein de la Mission requêtes et valorisation MRV créé en 2013 autour de 30 fonctionnaires est passé de quelques centaines en 2016 à plus de 101000 en 2020 (37 746 au SIE et 63595 au SIP) selon le rapport. En 2019, 22 \% des contrôles 
du SIE et $11 \%$ de ceux du SIP étaient dues à la MRV. La DGFIP s'est fixé un objectif de $50 \%$ des contrôles fiscaux en 2022.

\section{Politique fiscale}

Étude économique sur les effets de la réforme fiscale de 2018 (IFI,PFU,CSG)

Une étude INSEE/DRESS parue le 7 septembre évalue les effets sur les inégalités de niveau de vie et les finances publiques de la transformation de I'ISF en IFI et de la mise en place du PFU à $30 \%$ sur les revenus du patrimoine en France par la loi n²017-1837 du 30 décembre 2017 de finances 2018. Selon cette étude, la politique fiscale du président Macron favorise les foyers fiscaux les plus aisés, même si les gains sont limités par la hausse de la CSG, et le coût budgétaire de ces mesures fiscales (2,9 Md€) serait inférieur à celui anticipé par France Stratégie (5,1 Md€, v. Repères oct. 2019). L'étude prend en compte les effets comportementaux : les ventes de biens immobiliers de 340000 foyers fiscaux qui correspondent à une financiarisation du capital et la concentration des gains du PFU sur les $5 \%$ de foyers fiscaux les plus aisés tout en générant des recettes supplémentaires (410 M€ : hausse des dividendes avec I'IR, rapatriement d'exilés fiscaux avec I'IFI) et en creusant les inégalités par I'accentuation de la hausse de niveau de vie des ménages les plus aisés, avec un effet redistributif qui reste à évaluer...

Rapport sénatorial sur la fiscalité ultra-marine

La Délégation aux outre-mer du Sénat a publié un rapport d'information portant sur les enjeux financiers et fiscaux européens pour les outremer en 2020. Ce rapport formule 30 recommandations visant à mieux défendre les intérêts des territoires d'Outre-Mer sur le volet financier et fiscal. II rappelle la nécessité de reconduire les dispositifs fiscaux dérogatoires autorisés par l'UE en faveur des territoires d'Outre-mer qui arrivent à échéance le 31 décembre 2020 (octroi de mer, etc.).

\section{Évaluation du CICE : un nouveau rapport de France Stratégie}

France Stratégie publie un nouveau rapport d'évaluation du crédit d'impôt pour la compétitivité et l'emploi avec deux nouvelles études, reposant sur des méthodes différentes: une nouvelle analyse économétrique de données individuelles d'entreprises couvrant la période 2016-2017 et une analyse macro-économique complémentaire, permettraient d'estimer que près de 160000 emplois ont été créés ou sauvés, ce qui serait faible au regard du coût du CICE (18 Md€ en 2016). Le CICE ne semble pas avoir eu de résultats significatifs sur l'investissement des entreprises et n'a pas eu l'effet attendu par le législateur pour les entreprises industrielles, plus exposées à la concurrence internationale, et ayant une forte proportion de bas salaires, celles-ci étant plus réactives aux exonérations de cotisations sociales aux effets immédiats. L'effet sur l'emploi est toutefois plus net auprès des entreprises de services.

\section{$\lambda$ Impôt de production}

PLF 2021 : baisse des impôts de production, et I'autonomie fiscale locale?

La réforme de la fiscalité économique locale va connaître un nouvel épisode en loi de finances 2021 (art. 3 PLF 2021) après celui joué en loi de finances 2010 avec la suppression de la taxe professionnelle et son remplacement par la contribution économique territoriale (CVAE et CFE). Le dispositif gouvernemental (- $10 \mathrm{Mds} €$ au total) repose sur la suppression de la part CVAE des régions $(-7,25 \mathrm{Md} \epsilon)$, la réduction de moitié de la CFE (-1,75 Md€) et de la TFPB (- 1,54 Md€) à I'horizon 2022. Le législateur propose d'abaisser dès 2021 le taux de CVAE à hauteur de la part régionale (50\%) et d'y substituer une fraction de la TVA égale, en 2021, au montant de la CVAE perçu en 2020 ( y compris pour Mayotte, Corse, Martinique et Guyane). Le plafonnement de la CET en fonction de la valeur ajoutée est abaissé de $3 \%$ à $2 \%$. Fallait-il encore réduire la part contributive des entreprises aux ressources fiscales locales?

\section{$\pi$ Impôt sur les sociétés}

Taxe GAFA et sanctions américaines sur les produits français, statu quo?

L'article 1er de la loi du 24 juillet 2019 portant création d'une taxe sur les services numériques et modification de la trajectoire de baisse de I'IS a instauré une taxe sur certains services fournis par les grandes entreprises du secteur numérique, dite taxe GAFA (v. Repères, juillet 2019). En réponse, l'administration Trump a menacé de surtaxer " jusqu'à $100 \%$ » l'équivalent de $2,4 \mathrm{Mds} \$$ de produits français. Ont notamment été visées plusieurs filières françaises, telles que celle du champagne, plusieurs types de produits laitiers, les sacs à main en cuir, les cosmétiques, mais aussi la vaisselle en porcelaine ou encore les articles de cuisine en fonte. En janvier 2020, un accord a été trouvé autour d'un cadre global commun entre la France et les USA sur la taxa- 
tion digitale, et les efforts se poursuivent pour trouver un accord international à l'OCDE sur la taxation des entreprises numériques d'ici fin 2020 (v. Repères, janvier 2020). En vertu de cet accord, la France s'est engagée à suspendre jusqu'en décembre 2020, le prélèvement des acomptes dus au titre de la taxe sur les services numériques et les USA se sont engagés à ne pas imposer de sanctions sur les exportations françaises dans l'attente d'un accord à l'OCDE d'ici la fin de l'année.

\section{PLF 2021 : les principales mesures fiscales} concernant les entreprises

Le projet de loi de finances pour 2021 a été présenté en conseil des ministres le 28 septembre 2020. II reprend la plupart des mesures fiscales qui figurent dans le plan de relance, notamment la baisse des impôts locaux de production (suppression de la part régionale de la CVAE et ajustement du taux du plafonnement de la CET en fonction de la valeur ajoutée (art. 3, PLF 2021, cf. supra). Le législateur prévoit une exonération de CET en cas de création ou extension d'établissement (art. 42, PLF 2021) et une modernisation des paramètres de la méthode comptable d'évaluation servant au calcul des impôts fonciers (TFPB et CFE) de la plupart des établissements industriels, tout en neutralisant leurs effets sur le niveau des ressources des collectivités locales (art. 4, PLF 2021). Une autre mesure concerne la réévaluation des immobilisations corporelles et financières, tout en prévoyant une mesure temporaire de neutralisation des conséquences fiscales des réévaluations d'actifs (art. 5, PLF 2021). S'agissant de I'IS, une mesure temporaire prévoit l'étalement de la plus-value réalisée lors d'une opération de cession-bail d'immeuble par une entreprise (art. 39 novodecies du CGI). (du 28 sept. 2020 jusqu'au 31 déc. 2022).Le législateur souhaite supprimer progressivement la majoration de $25 \%$ des bénéfices des entreprises qui n'adhèrent pas à un organisme de gestion agréé soumis à un régime réel d'imposition à l'IR (art. 158-7-1 du CGI suite à art. 76 LFI 2006) (art. 7, PLF 2021). Le législateur introduit des aménagements au crédit d'impôt recherche (CIR) et au crédit d'impôt innovation (CII) dans un souci d'harmonisation européenne (art. 8, PLF 2021). En matière de TVA, une clarification des règles aux offres composites est proposée par le législateur. L'entrée en vigueur des règles modifiant le régime de TVA du commerce électronique issues du droit de l'UE (mesures adoptées par le Conseil de l'UE en déc. 2017 - «Paquet TVA sur le commerce électronique ") est reportée au 1 er juillet 2021 au lieu du 1 er janvier 2021 (art. 10, PLF 2021)). Le législateur met le régime de TVA des gains de course hippique en conformité avec le droit de I'UE (v. CJUE, 10 novembre 2016, Pavlina Bastova, affaire C-432/15) Par ailleurs, le législateur créé un régime de groupe de TVA (en transposant art. 11 de la directive «TVA » $n^{\circ}$ 2006/112/CE), et révise le champ du dispositif du groupement autonome de personnes (art. 45, PLF 2021). En matière de droits d'enregistrement, le caractère obligatoire de l'enregistrement de certains actes de sociétés est supprimé afin d'alléger les démarches des sociétés, notamment pour les actes de sociétés à très faible enjeu budgétaire (art. 18, PLF 2021). En matière de contributions environnementales et d'urbanisme diverses, la taxation de l'électricité est simplifiée (art. 13, PLF 2021), les taxes sur les véhicules à moteur sont refondues (art. 14, PLF 2021), les incitations à l'utilisation d'énergies renouvelables dans les transports sont renforcées (art. 15, PLF 2021) et la taxe d'aménagement est adaptée en vue de lutter contre l'artificialisation des sols (art. 43, PLF 2021). Le législateur prévoit le transfert de la gestion des taxes d'urbanisme à la DGFiP, au second semestre 2022, qui n'en assure aujourd'hui que le recouvrement (art. 44, PLF 2021). Enfin, le législateur supprime certaines taxes fiscales à faible rendement budgétaire (art. 16, PLF 2021) et des dépenses fiscales inefficientes (art. 17, PLF 2021). Au titre des mesures diverses, le taux de l'intérêt de retard et de l'intérêt moratoire (art. 55 de la loi de finances rectificative pour 2017), qui est de à 0,2\% par mois de retard, et ce jusqu'au 31 décembre 2020, est prorogé.

\section{$\lambda$ Fiscalité écologique}

\section{Deux propositions de loi fiscale "vertes "} d'exonération de TVA...

Deux propositions de loi ( $n^{\circ} 3325$ et $n^{\circ} 3326$ ) déposées à l'Assemblée nationale, suite aux constats de I'ADEME et du CITEPA, propose d'exonérer de TVA, d'une part, les travaux de rénovation énergétique afin de relever le défi climatique, sauver le secteur du bâtiment et ses emplois, et augmenter le pouvoir d'achat des ménages; et d'autre part, les prestations relatives aux transports de voyageurs, afin de rendre ces modes de transport plus compétitifs et d'inciter à les utiliser davantage.

\section{$\lambda$ Impôt sur le revenu}

\section{PLF 2021 : les principales mesures fiscales concernant les ménages}

Le projet de loi de finances pour 2021 a été présenté en conseil des ministres le 28 septembre 2020. Concernant les ménages, on retrouve 
I'indexation sur l'inflation (indice des prix à la consommation hors tabac de 2019 par rapport à 2018, soit 1 \%) du barème de l'IR pour les revenus 2020 et des grilles de taux par défaut du PAS, afin de contenir les effets de l'inflation sur le niveau d'imposition des ménages. Le barème de l'IR est ajusté en conséquences (art. 196 B du CGI) ainsi que le plafond du quotient familial (art. 197-II du CGI), le montant de l'abattement accordé en cas de rattachement d'un enfant majeur marié ou chargé de famille (art. 196 B du CGI), et les réductions d'impôt résultant de l'application du quotient familial qui sont réévaluées. Le législateur prévoit également, pour les revenus perçus ou réalisés à compter du $1{ }^{\text {er }}$ janvier 2021, d'ajuster les limites des tranches de revenus des grilles de taux par défaut du PAS en fonction de l'évolution du barème de I'IR (art. 2, PLF 2021). Ensuite, le législateur prévoit le maintien d'un crédit d'impôt en faveur de l'acquisition et de la pose de systèmes de charge pour véhicule électrique (art. 12, PLF 2021).

\section{Management public}

\section{$\pi$ Politique de modernisation}

Mieux conduire et partager les évaluations de politiques publiques

Le 3 septembre, le Conseil d'État a présenté son étude annuelle consacrée à l'évaluation des politiques publiques. Si cette démarche connaît un intérêt grandissant (2 600 évaluations entre 2007 et 2017) et si des progrès ont été réalisés, des faiblesses récurrentes demeurent : cloisonnement dans la programmation et dans la réalisation des travaux, caractère très institutionnel de l'évaluation en France qui n'associe pas suffisamment la recherche, anticipation et moyens parfois insuffisants, méthodologie pas toujours rigoureuse, association des citoyens trop rare, lacunes dans les domaines couverts, insuffisante publicité des travaux et de la prise en compte des résultats par les décideurs. Le Conseil d'État formule 20 recommandations autour de trois axes :se donner les moyens de mieux évaluer notamment en développant les commandes d'évaluation par le Parlement; améliorer la qualité des évaluations par un pilotage collégial et pluraliste, la prévention des conflits d'intérêt et une association des citoyens ; mieux diffuser les résultats des évaluations et les plans d'action qui en découlent.

\section{$\pi$ Administration numérique}

La feuille de route de la Dinum actualisée

La direction du numérique a publié une version actualisée de sa feuille de route Techgouv.
1 Md€ du plan de relance pour le numérique public

Selon la brochure du plan de relance publiée le 3 septembre, $1 \mathrm{Md} €$ est consacré au financement de l'innovation et de la transformation numériques de l'administration de l'État et des collectivités locales. Parmi les projets déjà fléchés : le nouveau poste de travail de l'agent public, la continuité pédagogique, un nouveau fonds de soutien à l'innovation et à la transformation numérique de l'État et des territoires, la cybersécurité et la future identification numérique régalienne. Dans un entretien publié par Les Echos du 10 septembre, la ministre de la fonction et de la transformation publiques annonce que l'objectif est de " faire en sorte que l'administration soit en phase avec le reste du pays et avec les entreprises qui seront de plus en plus numérisées ». $500 \mathrm{M} €$ du plan de relance seront utilisés pour des projets internes aux ministères et $500 \mathrm{M} €$ pour des fonds dédiés à des outils interministériels, à la transformation numérique et aux démarches en ligne. La ministre souhaite qu'en 2022, les 250 démarches les plus usuelles soient toutes accessibles en ligne avec un taux minimum de $70 \%$ de satisfaction des usagers.

\section{Modernisation de Légifrance}

Un décret $n^{\circ} 2020-1119$ du 8 septembre, publié au JORF du 10 septembre, texte $n^{\circ} 2$ modifie le décret du 7 août 2002 organisant un service public de diffusion du droit par internet pour prendre en compte la modernisation du site, effective à partir du 12 septembre :recherche facilitée sur l'ensemble des fonds documentaires, accès simplifié aux contenus, recherches multicritères, suivi des versions successives d'un texte, possibilité de réutilisation des données, numérisation des JO papiers depuis 1869. (Sur la version bêta, v. Repères d'octobre 2019).

\section{Un rapport du Sénat sur l'inclusion numé- rique}

Un rapport $n^{\circ} 711$, enregistré le 17 septembre, du Sénateur Raymond Vall, confirme le constat de l'illectronisme : 14 millions de Français ne maîtrisent pas le numérique et près d'un Français sur deux n'est pas à l'aise. En conséquence, "la dématérialisation à marche forcée des services publics laisserait sur le bord de la route trois Français sur cinq ».II constate que la stratégie nationale lancée en 2018 a été sous-dimensionnée et sous-financée. II formule 45 propositions pour une politique ambitieuse d'inclusion numérique dotée de 1 Md€. 


\section{Administration centrale}

\section{Création du Haut commissaire au Plan}

Un décret $n^{\circ}$ 2020-1101 du $1^{\text {er }}$ septembre 2020,publié au JORF du 2 septembre,texte $n^{\circ} 1$ institue un Haut commissaire au Plan «chargé d'animer et de coordonner les travaux de planification et de réflexion prospective conduits pour le compte de l'État et d'éclairer les choix des pouvoirs publics au regard des enjeux démographiques, économiques, sociaux, environnementaux, sanitaires, technologiques et culturels ». Un décret rectificatif publié au JORF du $3 \mathrm{sep}$ tembre, texte $n^{\circ} 1$, précise que le Haut Commissaire dispose: "du concours de France stratégie et des administrations et services de l'État susceptibles de contribuer à l'exercice de sa mission ». France Stratégie, créé par un décret du 22 avril 2013, avait succédé au Conseil d'analyse stratégique qui avait lui-même pris la suite du Commissariat général du Plan en 2006. Dans sa lettre de mission du 21 septembre, le Président de la République charge le Haut commissaire de "prendre en charge le long terme ».Dans son discours du 22 septembre devant le Conseil économique, social et environnemental, François Bayrou, a évoqué trois grands thèmes de travail: la « vitalité de la France » (écologie, démographie, identité, francophonie, recherche et innovation), "I'indépendance » (relocalisations, productions vitales, souveraineté agricole, dette.) et la « justice " (inégalités, éducation, santé, aménagement du territoire, aide au développement).

\section{$\pi$ Administration territoriale de l'État}

Fonctions et désignation des sous-préfets à la relance

Une circulaire du Premier ministre du $10 \mathrm{sep}$ tembre indique que les sous-préfets à la relance auront vocation à exercer des missions à forts enjeux et à piloter des projets complexes, en lien avec les chantiers de transformation lancés par le Gouvernement, les conséquences de la crise sanitaire et la relance de l'économie. Ils seront affectés, pour un an ou deux, dans des territoires de tous niveaux (de la région à l'arrondissement, en fonction des besoins) pour accompagner le plan de relance et lever les blocages éventuels.....lls seront désignés parmi les corps relevant du dispositif des « missions prioritaires de l'État» (énarques ou polytechniciens sortis dans les grands corps) et plus largement au sein d'autres corps auxquels ouvre l'Ena ou de corps comparables. Une procédure de sélection est fixée pour aboutir à une prise de poste au 1er janvier 2021.(v.aussi Repères de août 2020).

\section{Annonce des premières relocalisations à la} DGFiP

Le 30 septembre, le ministre chargé des comptes publics a réuni les 50 maires des villes moyennes concernées par la première vague de relocalisation (ou « démétropolisation ») de 2500 emplois pour annoncer le détail et les modalités des opérations ( $v$. le dossier de presse). Les services concernés n'auront pas de contact directs avec le public mais seront des services de soutien ou d'appui qui travailleront à distance (" back office ») tels que des centres de renseignements téléphonés, des service d'appui à la publicité foncière, des antennes de services des impôts des entreprises implantés dans les grands villes, des pôles d'appui au contrôle fiscal, des centres de contact et de gestion des paies et des retraites. La mise en place se fera progressivement sur la base du volontariat jusqu'en 2024. Une liste d'une quinzaine d'autres communes devrait être prochainement annoncée.

\section{$\pi$ Fonction publique}

\section{L'organisation du travail dans la fonction publique avec la Covid 19}

Une circulaire du Premier ministre 1er septembre précise les règles de prise en compte de l'épidémie de covid-19 dans la fonction publique de I'État : port du masque obligatoire ; télétravail favorisé ; isolement en cas de maladies ou de symptômes.

La ministre consulte les fonctionnaires et donne ses premières orientations

La nouvelle ministre de la transformation et de la fonction publiques, Amélie de Montchanin, a adressé, le 4 septembre, une lettre aux « chers agents publics » pour reconnaître et encourager leur investissement dans la lutte contre la Covid et pour le plan de relance. Elle lance un appel à propositions de mesures à prendre à deux niveaux : le ministère et la vie quotidienne de l'agent. Dans plusieurs entretiens avec la presse, la ministre a notamment insisté sur l'administration numérique dans le plan de relance (v. supra), sur la simplification de l'action publique, sur l'égalité des chances dans l'accès aux postes de responsabilité de la haute fonction publique, sur la diversité : «Ceux qui administrent le pays doivent ressembler aux Françaises et aux Français ", sur la mobilité et sur le regroupement sous une même autorité de la direction du numérique, de la direction interministérielle de la transformation publique et de la direction générale de l'administration et de la fonction publique. 
La Cour des comptes recommande de clarifier la gestion des contractuels

Un rapport de la Cour des comptes, rendu public le 22 septembre, constate l'augmentation rapide du nombre de contractuels dans la fonction publique (plus d'1 1 million, soit $18,4 \%$ des effectifs) et une rigidification progressive des règles de gestion. Au moment où les possibilités de recrutement sont facilitées en application de la loi du 6 août 2018 de transformation de la fonction publique, la Cour recommande de développer le recrutement des agents publics sur titres ou par validation des acquis de l'expérience, de mettre en place des référentiels de rémunération des contractuels pour les métiers en tension, de proposer à la négociation collective des cadres de gestion des contractuels, d'envisager l'affiliation des employeurs publics aux régimes d'assurance-chômage, d'améliorer le dialogue social.

\section{Ministère des finances}

Un rapport de la Cour des comptes sur la DGDDI

Le 24 septembre, la Cour des comptes a rendu public un rapport sur la direction générale des douanes et des droits indirects qui regroupe 17000 agents dans 674 implantations pour l'exercice de trois missions principales : la surveillance et la lutte contre les fraudes et trafics, le soutien à l'activité économique, le recouvrement de certains droits et contributions indirects et, accessoirement, la participation à la gestion des flux migratoires, à l'action de l'État en mer et à I'accompagnement des filières vitivinicoles et du commerce du tabac. Malgré des efforts d'adaptation dans le contexte européen, la Cour relève diverses rigidités et insuffisances dans la gestion et recommande un recentrage des activités autour de la gestion des flux de marchandises et de personnes.

V.aussi Fiscalité.

\section{EUROPE}

\section{入 Pacte de stabilité}

Rapport de la Cour des comptes sur le semestre européen

Le 3 septembre 2020, la Cour des comptes européenne a publié un rapport spécial 16/2020 consacré au Semestre européen. Elle observe que les recommandations par pays abordent des problématiques importantes, mais que leur mise en œuvre laisse à désirer.

\section{$\pi$ Budget européen}

Budget de I'UE pour 2021: le Conseil arrête sa position

Le 9 septembre 2020, les ambassadeurs auprès de I'UE ont approuvé la position du Conseil sur le projet de budget de I'UE pour 2021. Au total, la position du Conseil sur le budget du prochain exercice prévoit 162,9 milliards $€$ en crédits d'engagement et 164,8 milliards $€$ en crédits de paiement. Le budget 2021 sera complété par des actions visant à soutenir la reprise post-COVID-19, qui seront financées par Next Generation EU, le plan de relance de I'UE pour faire face à la pandémie, doté d'une enveloppe de 750 milliards d'euros. II s'agit du premier budget annuel au titre du cadre financier pluriannuel pour la période 2021-2027 (CFP). Étant donné que les négociations trilatérales avec le Parlement sur le prochain CFP sont en cours, le Conseil réévaluera sa position à la lumière du texte final du CFP et de la lettre rectificative attendue de la Commission à un stade ultérieur de la procédure.

\section{Budget de l'Union et état de droit}

Le 30 septembre 2020, les ambassadeurs des États membres auprès de l'UE ont approuvé ce jour la position du Conseil sur un règlement relatif à un régime général de conditionnalité pour la protection du budget de l'Union. Le 2 mai 2018, la Commission avait présenté une proposition de règlement relatif à la protection du budget de I'Union en cas de défaillance généralisée de l'état de droit dans un État membre, dans le cadre d'un ensemble de propositions relatives au cadre financier pluriannuel pour la période 2021-2027. Malgré les oppositions de la Hongrie, la position du Conseil a obtenu le soutien de la majorité qualifiée des États membres. L'accord intervenu va permettre l'ouverture de négociations avec le Parlement européen sur la proposition.

\section{$\pi$ Euro}

Inquiétudes face à l'appréciation de l'euro par rapport au dollar

Lors de sa conférence de presse de rentrée jeudi 10 septembre, Christine Lagarde a fait état de ses préoccupations par rapport à l'évolution de l'euro tout en réaffirmant que la $B C E$ restait prête à prendre de nouvelles mesures pour sortir la zone euro de la récession. Sa prévision d'inflation reste inchangée à $0,3 \%$ pour 2020 , malgré le passage du taux d'inflation dans la zone euro en territoire négatif en août, du jamais vu depuis mai 2016. L'évolution la plus commentée est l'appréciation de la monnaie unique par rapport à la de- 
vise américaine. L'euro a franchi une semaine auparavant le seuil de 1,20 dollar pour la première fois depuis 2018. Cela s'explique par différents facteurs, dont la modification de la stratégie de la Réserve fédérale américaine et l'accord de juillet entre les États de l'Union européenne sur un plan de relance de 750 milliards d'euros.

Un membre du directoire de la BCE prend ses distances

Dans un entretien paru le 23 septembre sur le site Bloomberg, Yves Mersch, ancien gouverneur de la banque centrale luxembourgeoise et membre du directoire de la Banque centrale européenne depuis 2012, a rappelé que les mesures exceptionnelles prises par la BCE depuis 2010 de rachat d'actifs pour soutenir la croissance devaient rester provisoires. Plusieurs membres de la BCE exposent ainsi des points de vue divergents dont Mme Lagarde a déclaré ne pas être inquiète le 28 septembre lors de son audition trimestrielle devant le Parlement européen.

\section{入 États-membres}

Plaidoyer proeuropéen du président de la banque centrale néerlandaise

Lors d'une conférence donnée le 1er septembre 2020 et largement remarquée par la presse, le président de la banque centrale Klaas Knot a livré un plaidoyer pro-européen inattendu, en contradiction avec les positions du Premier ministre néerlandais Mark Rutte, I'un des leaders des États européens "frugaux ». II s'est notamment prononcé en faveur de mesures allant au-delà de I'accord difficilement atteint lors du sommet extraordinaire de juillet 2020.

\section{Projet de taxe what's app en Espagne}

Le 14 septembre la presse a fait état d'un projet du gouvernement espagnol de faire adopter l'année prochaine une loi qualifiant les applications de messagerie instantanée telles WhatsApp ou Telegram, d'opérateurs téléphoniques. Elles seront taxées sur leurs revenus issus des services de messagerie, à partir d'un million d'euros de recettes. Ce projet est motivé par le souci d'égaliser la concurrence dans ce secteur entre ces messageries et les opérateurs téléphoniques traditionnels.

\section{INTERNATIONAL}

\section{入 Relations multilatérales}

\section{Candidature américaine à I'OCDE}

Le 2 septembre 2020, la Maison-Blanche annonçait la candidature de Chris Liddell, un des proches conseillers de Donald Trump, pour le poste de secrétaire général de l'OCDE. La succession du mexicain Angel Gurría est devenue stratégique en raison du "leadership » assumé par l'organisation dans la révision mondiale de la fiscalité des géants du numérique que les ÉtatsUnis veulent freiner.

\section{Fiscalité}

OCDE : nouvelle édition du rapport « Tax policy réforms ॥

Le 3 septembre I'OCDE a publié la $5^{\text {e édition }}$ de son rapport "Tax policy reforms » (en anglais seulement), décrivant les tendances des réformes fiscales dans les pays de l'OCDE et certaines économies partenaires (Argentine, Indonésie, Afrique du Sud, mais aussi Chine). II s'intéresse particulièrement au rôle des réformes fiscales pour répondre rapidement aux effets économiques de la crise sanitaire actuelle.

Aurélien BAUDU (fiscalité et procédure fiscale), Fabrice BIN (Europe, international), Jean-François CALMETTE (budget de l'État et des opérateurs),

Matthieu HOUSER (finances locales), Michel LE CLAINCHE (comptabilité publique, management public, coordination), Yves TERRASSE (finances sociales).

Retrouvez sur www.gestionfinancespubliques. info :

- I'intégralité des REPÈRES d'actualité des finances et de la gestion publiques depuis 2015 -le 15 du mois, les REPĖRES d'actualité du mois précédent

-le 15 janvier 2020, le BEST OF des repères d'actualité de l'année 2019.

- et, en continu, les réflexions des blogueurs de G\&FP. 